\title{
HESS very-high-energy gamma-ray sources without identified counterparts
}

\author{
F. Aharonian ${ }^{1,13}$, A. G. Akhperjanian ${ }^{2}$, U. Barres de Almeida ${ }^{8, \star}$, A. R. Bazer-Bachi ${ }^{3}$, B. Behera ${ }^{14}$, M. Beilicke ${ }^{4}$,
} W. Benbow ${ }^{1}$, K. Bernlöhrr ${ }^{1,5}$, C. Boisson ${ }^{6}$, O. Bolz ${ }^{1}$, V. Borrel ${ }^{3}$, I. Braun ${ }^{1}$, E. Brion ${ }^{7}$, A. M. Brown ${ }^{8}$, R. Bühler ${ }^{1}$, T. Bulik ${ }^{24}$, I. Büsching, , T. Boutelier ${ }^{17}$, S. Carrigan ${ }^{1}$, P. M. Chadwick ${ }^{8}$, L.-M. Chounet ${ }^{10}$, A. C. Clapson ${ }^{1}$, G. Coignet ${ }^{11}$, R. Cornils ${ }^{4}$, L. Costamante ${ }^{1,28}$, M. Dalton ${ }^{5}$, B. Degrange ${ }^{10}$, H. J. Dickinson ${ }^{8}$, A. Djannati-Ataï ${ }^{12}$, W. Domainko ${ }^{1}$, L.O'C. Drury ${ }^{13}$, F. Dubois ${ }^{11}$, G. Dubus ${ }^{17}$, J. Dyks ${ }^{24}$, K. Egberts ${ }^{1}$, D. Emmanoulopoulos ${ }^{14}$, P. Espigat ${ }^{12}$, C. Farnier ${ }^{15}$, F. Feinstein ${ }^{15}$, A. Fiasson ${ }^{15}$, A. Förster ${ }^{1}$, G. Fontaine ${ }^{10}$, Seb. Funk ${ }^{5}$, M. Füßling ${ }^{5}$, Y. A. Gallant ${ }^{15}$, B. Giebels ${ }^{10}$, J. F. Glicenstein ${ }^{7}$, B. Glück ${ }^{16}$, P. Goret ${ }^{7}$, C. Hadjichristidis ${ }^{8}$, D. Hauser ${ }^{1}$, M. Hauser ${ }^{14}$, G. Heinzelmann ${ }^{4}$, G. Henri ${ }^{17}$, G. Hermann ${ }^{1}$, J. A. Hinton ${ }^{25}$, A. Hoffmann ${ }^{18}$, W. Hofmann ${ }^{1}$, M. Holleran ${ }^{9}$, S. Hoppe ${ }^{1}$, D. Horns ${ }^{18}$, A. Jacholkowska ${ }^{15}$, O. C. de Jager ${ }^{9}$, I. Jung ${ }^{16}$, K. Katarzyński2 ${ }^{27}$, E. Kendziorra ${ }^{18}$, M. Kerschhaggl ${ }^{5}$, B. Khélifi ${ }^{10}$, D. Keogh ${ }^{8}$, Nu. Komin ${ }^{15}$, K. Kosack ${ }^{1}$, G. Lamanna ${ }^{11}$, I. J. Latham ${ }^{8}$, A. Lemière ${ }^{12}$, M. Lemoine-Goumard ${ }^{10}$, J.-P. Lenain ${ }^{6}$, T. Lohse ${ }^{5}$, J. M. Martin ${ }^{6}$, O. Martineau-Huynh ${ }^{19}$, A. Marcowith ${ }^{15}$, C. Masterson ${ }^{13}$, D. Maurin ${ }^{19}$, G. Maurin ${ }^{12}$, T. J. L. McComb ${ }^{8}$, R. Moderski ${ }^{24}$, E. Moulin ${ }^{7}$, M. de Naurois ${ }^{19}$, D. Nedbal ${ }^{20}$, S. J. Nolan ${ }^{8}$, S. Ohm ${ }^{1}$, J.-P. Olive ${ }^{3}$, E. de Oña Wilhelmi ${ }^{12}$, K. J. Orford ${ }^{8}$, J. L. Osborne ${ }^{8}$, M. Ostrowski ${ }^{23}$, M. Panter ${ }^{1}$, G. Pedaletti ${ }^{14}$, G. Pelletier ${ }^{17}$, P.-O. Petrucci ${ }^{17}$, S. Pita ${ }^{12}$, G. Pühlhofer ${ }^{14}$, M. Punch ${ }^{12}$, S. Ranchon ${ }^{11}$, B. C. Raubenheimer ${ }^{9}$, M. Raue ${ }^{4}$, S. M. Rayner ${ }^{8}$, M. Renaud ${ }^{1}$, J. Ripken ${ }^{4}$, L. Rob ${ }^{20}$, L. Rolland ${ }^{7}$, S. Rosier-Lees ${ }^{11}$, G. Rowell ${ }^{26}$, B. Rudak ${ }^{24}$, J. Ruppel ${ }^{21}$, V. Sahakian ${ }^{2}$, A. Santangelo ${ }^{18}$, R. Schlickeiser ${ }^{21}$, F. Schöck ${ }^{16}$, R. Schröder ${ }^{21}$, U. Schwanke ${ }^{5}$, S. Schwarzburg ${ }^{18}$, S. Schwemmer ${ }^{14}$, A. Shalchi ${ }^{21}$, H. Sol ${ }^{6}$, D. Spangler ${ }^{8}$, . Stawarz $^{23}$, R. Steenkamp ${ }^{22}$, C. Stegmann ${ }^{16}$, G. Superina ${ }^{10}$, P. H. Tam ${ }^{14}$, J.-P. Tavernet ${ }^{19}$, R. Terrier ${ }^{12}$, C. van Eldik $^{1}$, G. Vasileiadis ${ }^{15}$, C. Venter ${ }^{9}$, J. P. Vialle ${ }^{11}$, P. Vincent ${ }^{19}$, M. Vivier ${ }^{7}$, H. J. Völk ${ }^{1}$, F. Volpe ${ }^{10}$, S. J. Wagner ${ }^{14}$, M. Ward ${ }^{8}$, A. A. Zdziarskii ${ }^{24}$, and A. Zech ${ }^{6}$

(Affiliations can be found after the references)

Received 20 August 2007 / Accepted 2 October 2007

\section{ABSTRACT}

Context. The detection of gamma rays in the very-high-energy (VHE) energy range ( $100 \mathrm{GeV}-100 \mathrm{TeV})$ provides a direct view of the parent population of ultra-relativistic particles found in astrophysical sources. For this reason, VHE gamma rays are useful for understanding the underlying astrophysical processes in non-thermal sources.

Aims. We investigate unidentified VHE gamma-ray sources that have been discovered with HESS in the most sensitive blind survey of the Galactic plane at VHE energies conducted so far.

Methods. The HESS array of imaging atmospheric Cherenkov telescopes (IACTs) has a high sensitivity compared with previous instruments ( $\sim 0.01 \mathrm{Crab}$ in $25 \mathrm{~h}$ observation time for a $5 \sigma$ point-source detection), and with its large field of view, is well suited for scan-based observations. The on-going HESS survey of the inner Galaxy has revealed a large number of new VHE sources, and for each we attempt to associate the VHE emission with multi-wavelength data in the radio through X-ray wavebands.

Results. For each of the eight unidentified VHE sources considered here, we present the energy spectra and sky maps of the sources and their environment. The VHE morphology is compared with available multi-wavelength data (mainly radio and X-rays). No plausible counterparts are found.

Key words. gamma rays: observations - Galaxy: general - cosmic rays - surveys

\section{Introduction}

VHE gamma-ray astronomy has recently entered an new era of discovery with the introduction of the latest generation Imaging Atmospheric Cherenkov Telescopes (IACTs) such as HESS (the High Energy Stereoscopic System). Since HESS began operation in 2004, about two dozen new VHE sources have been revealed. Presently identified VHE gamma-ray sources belong to one of four categories: active galactic nuclei (AGN), pulsar wind nebulae (PWN), shell-type supernova remnants (SNR), or X-ray binaries (XRB); recently also VHE emission was detected which

* Supported by CAPES Foundation, Ministry of Education of Brazil. may be associated with a young stellar cluster (Aharonian et al 2007c). All these identified source classes also exhibit emission in the radio and/or X-ray regime. However, several VHE sources discovered by HESS in the field-of-view of other known sources (Aharonian et al. 2005c) or during the HESS Galactic plane survey (Aharonian et al. 2005b, 2006d) have not been identified with objects from which VHE emission is expected. The first unidentified VHE source was TeV J2032+4130 (Aharonian et al. 2002, 2005a), which was discovered by the HEGRA IACT system. HESS J1303-631 (Aharonian et al. 2005c) was found in the field-of-view of the binary pulsar system PSR B1259-63/SS 2883, and several other sources were subsequently 
discovered in the Galactic plane survey. To date, these objects remain unidentified; HESS J1303-631 has even been postulated to be related to such an exotic phenomenon as a gamma-ray burst remnant (Atoyan et al. 2006).

VHE gamma rays are tracers of non-thermal particle acceleration, and their production can be explained by the presence of either high-energy electrons or protons. In electron scenarios, gamma rays are primarily produced by inverse-Compton up-scattering of background photon fields by high-energy electrons. Significant X-ray and radio emission is predicted since the same population of electrons should emit synchrotron radiation at longer wavelengths. For typical Galactic magnetic field strengths, the energy flux of the X-ray component of the photon spectrum in the $\mathrm{keV}$ range is predicted to be comparable to the energy flux in the TeV range. The X-ray component of the spectrum may be suppressed, however, if there is a cutoff in the parent electron spectrum below $\sim 10 \mathrm{TeV}$ (Aharonian et al. 1997). In proton scenarios, VHE gamma rays are produced primarily from the decay of neutral pions $\left(\pi^{0}\right)$ that result from proton-proton interactions. If gamma rays are produced only via $\pi^{0}$ decay, a strong X-ray or radio signal may not be present; however, proton interactions also produce charged pions and cascades of secondary electrons that should generate a continuum of X-ray and radio synchrotron emission. Since it is difficult to explain VHE gamma-ray emission without at least a weak lower-energy counterpart, the lack of low-energy emission from the unidentified HESS sources puts significant constraints on physical conditions and/or particle acceleration processes in their sources. While the explanation may simply be that sufficiently deep multi-wavelength observations of the objects have not yet been made, the possibility exists that there is a new class of object that does not follow the predictions of standard emission models.

Recent observations of the Galactic plane and further reobservations of known sources with HESS have allowed for the study of some of the weaker Galactic sources at increased sensitivity and have revealed new VHE gamma-ray sources in addition to those described by Aharonian et al. (2006d). Similar to the previously mentioned objects, several of these sources have no obvious cataloged counterpart at longer wavelengths, and consequently their emission mechanism is unidentified. In this paper, we focus on eight VHE emitters without obvious counterpart that have been detected by HESS. Of these sources, an updated analysis is given for two previously published unidentified sources for which subsequent observations have provided significantly better statistics, and the detections of six new unidentified sources are reported. New VHE detections within the Galactic plane of known objects (PWN, SNRs, etc.) have been or will be reported elsewhere (e.g. in Aharonian et al. 2007a,b,c).

\section{Observations and technique}

\subsection{The HESS Instrument}

HESS (the High Energy Stereoscopic System) is an array of four atmospheric Cherenkov telescopes located in the Khomas highland of Namibia at an altitude of $1800 \mathrm{~m}$ above sealevel. Each telescope consists of a $107 \mathrm{~m}^{2}$ optical reflector made up of segmented mirrors that focus light into a camera of 960 photo-multiplier tube pixels (Bernlöhr et al. 2003). The telescopes image the UV/blue flashes of Cherenkov light emitted by the secondary particles produced in gamma-rayinduced air-showers. Stereoscopic shower observations using the imaging atmospheric Cherenkov technique (e.g. Hillas 1996;
Weekes 1996; Daum et al. 1997) allow for accurate reconstruction of the direction and energy of the primary gamma rays as well as for the rejection of background events from air showers of cosmic ray origin. HESS is sensitive to gamma rays above a post-cuts threshold energy of approximately $150 \mathrm{GeV}$ and has an average energy resolution of $\sim 16 \%$ (Aharonian et al. 2006b). Additionally, the high angular resolution $\left(\sim 0.1^{\circ}\right)$, large field-ofview $\left(\sim 5^{\circ}\right)$, and good off-axis sensitivity of the HESS array make it well suited for extended sources and scan-based observations, where the source position is not known a priori.

\subsection{Data}

The observations discussed here were taken as part of the ongoing HESS Galactic plane survey which currently covers the band $-50^{\circ}<l<60^{\circ}$ in galactic longitude and $-3^{\circ}<b<3^{\circ}$ in latitude. Data were taken as a series of 28-min observations (runs) centered on regular grid points covering the survey area. Additionally, several established sources were observed with pointed follow-up observations in wobble mode, where data are taken with an alternating offset from the target position of typically $\pm 0.7^{\circ}$ in right ascension or declination. The set of usable runs were selected based on a standard set of hardware and weather conditions (Aharonian et al. 2006b). The sources in this study were chosen by selecting all locations in the HESS Galactic plane scan data set that have a pre-trials detection significance (with a fixed integration radius of $0.22^{\circ}$ ) greater than $6 \sigma$ (corresponding to a post-trials significance of $4 \sigma$, based on the very conservative estimate for the number of trials given in Aharonian et al. 2006d), and for which no obvious cataloged counterpart can be associated (based on the criteria given in Sect. 2.4). Sources that were previously published (e.g. in Aharonian et al. 2006d) were excluded, except those that have had increases in significance over $3 \sigma$ due to subsequent observation. The eight sources that pass these selection criteria and their center positions (based on a model fit described in Sect. 2.3) are summarized in Table 1. For reference, a summary of published results on previously reported unidentified VHE objects is given in Table 2.

\subsection{Analysis technique}

The data presented here were analyzed using the standard HESS analysis scheme: calibrations are applied to the raw shower images (Aharonian et al. 2004) followed by an image cleaning procedure which removes noise due to fluctuations in the optical night-sky background light. The images are then parametrized using the Hillas moment-analysis technique (Hillas 1996), and gamma-ray selection criteria based on the image parameters are applied (Aharonian et al. 2006b). To reduce systematic effects in the spectrum due to off-axis sensitivity that arise when images fall near the camera edge, an additional cut is applied to accept only data runs which are taken within an angular distance $\psi$ from the respective position of the object under analysis. For the spectral analysis, this is conservatively set to $2.0^{\circ}$ to minimize systematic errors on the energy estimates (providing an average offset of $1.0^{\circ} \pm 0.1^{\circ}$ ), while for the generation of the sky maps, it was set to $2.5^{\circ}$ to maximize the number of photons detected (giving an average offset of $1.9^{\circ} \pm 0.2^{\circ}$ ). Images from events passing the cuts for each telescope are combined to reconstruct the shower direction and energy. In the data presented here, two sets of gamma-ray selection criteria are used to suppress events with hadronic origin: standard cuts, which are 
Table 1. Positions in equatorial (J2000 epoch) and Galactic $(l, b)$ coordinates along with the detection significances of unidentified sources in the HESS Galactic Plane scan discussed in this paper. $S$ is the significance (number of standard deviations above the background level) of the source using a fixed integration radius of $0.22^{\circ}$, which was used for selecting the sources from the scan data. The position of each source is based on a model fit to the background-subtracted gamma-ray maps (discussed in Sect. 2.3 and Table 3). The fit positions have an average statistical error of 0.05 degrees. Sources marked with a $†$ are previously published in Aharonian et al. (2006d) and have been updated with new data. The exposure time is corrected for the off-axis sensitivity of the telescope system and accounts for instrumental readout dead-time.

\begin{tabular}{lccccccc}
\hline \hline Source & Right Ascension & Declination & $l\left(^{\circ}\right)$ & $b\left(^{\circ}\right)$ & Time $(\mathrm{h})$ & $S(\sigma)$ & Excess (cts) \\
\hline HESS J1427-608 & $14^{\mathrm{h}} 27^{\mathrm{m}} 52^{\mathrm{s}}$ & $-60^{\circ} 51^{\prime} 00^{\prime \prime}$ & 314.409 & -0.145 & 21 & 7.3 & 197 \\
HESS J1626-490 & $16^{\mathrm{h}} 26^{\mathrm{m}} 04^{\mathrm{s}}$ & $-49^{\circ} 05^{\prime} 13^{\prime \prime}$ & 334.772 & 0.045 & 12 & 7.5 & 153 \\
HESS J1702-420† & $17^{\mathrm{h}} 02^{\mathrm{m}} 44^{\mathrm{s}}$ & $-42^{\circ} 00^{\prime} 57^{\prime \prime}$ & 344.304 & -0.184 & 9 & 12.8 & 412 \\
HESS J1708-410 & $17^{\mathrm{h}} 08^{\mathrm{m}} 24^{\mathrm{s}}$ & $-41^{\circ} 05^{\prime} 24^{\prime \prime}$ & 345.683 & -0.469 & 39 & 10.7 & 513 \\
HESS J1731-347 & $17^{\mathrm{h}} 31^{\mathrm{m}} 55^{\mathrm{s}}$ & $-34^{\circ} 42^{\prime} 36^{\prime \prime}$ & 353.565 & -0.622 & 14 & 8.1 & 218 \\
HESS J1841-055 & $18^{\mathrm{h}} 40^{\mathrm{m}} 55^{\mathrm{s}}$ & $-05^{\circ} 33^{\prime} 00^{\prime \prime}$ & 26.795 & -0.197 & 26 & 10.6 & 346 \\
HESS J1857+026 & $18^{\mathrm{h}} 57^{\mathrm{m}} 11^{\mathrm{s}}$ & $02^{\circ} 40^{\prime} 00^{\prime \prime}$ & 35.972 & -0.056 & 21 & 8.7 & 223 \\
HESS J1858+020 & $18^{\mathrm{h}} 58^{\mathrm{m}} 20^{\mathrm{s}}$ & $02^{\circ} 05^{\prime} 24^{\prime \prime}$ & 35.578 & -0.581 & 25 & 7.0 & 168 \\
\hline
\end{tabular}

Table 2. Previously published unidentified VHE sources, not discussed in this paper. Coordinates are in $\mathrm{J} 2000$ epoch, $\sigma_{\text {src }}$ is the intrinsic source extent (taking into account the instrumental response). Sources with $\ddagger$ have no obvious longer-wavelength counterpart. HESS J1632478 has a possible HMXB counterpart, but the VHE source is extended; HESS J1634-472 may be related to an unidentified INTEGRAL source or nearby SNR, but is offset and morphologically dissimilar; HESS J1745-303 is partially coincident with an unidentified EGRET source; and HESS J1837-069 is coincident with an as yet unidentified ASCA source. Results are from Aharonian et al. (2005c), Aharonian et al. (2006d), and Aharonian et al. (2005a).

\begin{tabular}{lccc}
\hline \hline Source & RA & Dec & $\sigma_{\text {src }}\left({ }^{\prime}\right)$ \\
\hline HESS J1303-631 & $13^{\mathrm{h}} 03^{\mathrm{m}} 00^{\mathrm{s}}$ & $-63^{\circ} 11^{\prime} 55^{\prime \prime}$ & 9.6 \\
HESS J1614-518 $\ddagger$ & $16^{\mathrm{h}} 14^{\mathrm{m}} 19^{\mathrm{s}}$ & $-51^{\circ} 49^{\prime} 12^{\prime \prime}$ & 13.8 \\
HESS J1632-478 & $16^{\mathrm{h}} 32^{\mathrm{m}} 09^{\mathrm{s}}$ & $-47^{\circ} 49^{\prime} 12^{\prime \prime}$ & 12.0 \\
HESS J1634-472 & $16^{\mathrm{h}} 34^{\mathrm{m}} 58^{\mathrm{s}}$ & $-47^{\circ} 16^{\prime} 12^{\prime \prime}$ & 6.6 \\
HESS J1745-303 & $17^{\mathrm{h}} 45^{\mathrm{m}} 02^{\mathrm{s}}$ & $30^{\circ} 22^{\prime} 12^{\prime \prime}$ & 12.6 \\
HESS J1837-069 & $18^{\mathrm{h}} 37^{\mathrm{m}} 38^{\mathrm{s}}$ & $-6^{\circ} 57^{\prime} 00^{\prime \prime}$ & 7.2 \\
TeV J2032+4130 & $20^{\mathrm{h}} 32^{\mathrm{m}} 57^{\mathrm{s}}$ & $41^{\circ} 29^{\prime} 57^{\prime \prime}$ & 6.2 \\
\hline
\end{tabular}

optimized using a simulated source with an energy spectrum with photon index $\Gamma=2.6$ and a flux that is $10 \%$ of the Crab Nebula (a standard bright gamma-ray source) at VHE energies, and hard cuts which are optimized for a harder spectrum source $(\Gamma=2.0)$ with a flux that is $1 \%$ of the Crab Nebula. Standard cuts have an intrinsically lower energy threshold, but are looser and accept more background events, while the hard cuts provide better gamma-hadron separation, and thus higher signalto-noise ratio, at the expense of an increased energy threshold. Unless otherwise noted, hard cuts are employed for the spectral and morphological analyses presented in this article since they provide smaller systematic errors due to a higher analysis energy threshold and better background rejection, though both sets are applied to check for consistency.

The sky maps used for determining the source location and morphology are generated by accumulating the points of origin of each gamma-ray candidate in a two-dimensional histogram, subtracting a background map modeled by counting the number of events which fall within an annulus (of average radius $0.5^{\circ}$ ) about each grid point, excluding emission regions (the ringbackground model described in Berge et al. 2007). The background is corrected for acceptance variations across the field of view. As an additional check, a background model using the radial gamma-ray acceptance profile (as determined by dedicated off-source observations and simulations) in the field of view of each run is also used and compared for consistency. An elongated two-dimensional Gaussian convolved with the HESS point-spread function is fit to the resulting excess map to determine the centroid position, position angle, and extent of the source. To define the full extent of the source for spectral analysis, a histogram of the squared distance of each event to the fit position $\left(\theta^{2}\right)$ is generated. The statistical significances of each excess measurement are calculated from the measured number of on- and off-source (background) events following the likelihood ratio procedure outlined in Li \& Ma (1983).

The background for spectra is estimated using the reflectedregion technique where background events are selected from circular off-source regions in the field of view that have the same angular size and offset from the observation center position as the on-source region (Aharonian et al. 2006b). Background regions containing other known sources are excluded. This technique provides a more accurate estimation of the background than the field-of-view model (described above) used to generate the sky maps, but is not as well suited for the generation of two-dimensional images.

Spectra are generated following Aharonian et al. (2006b) for all events that fall within an angular distance $\theta_{\text {int }}$ of the target position. This radius is chosen for each source as the distance where the radial excess distribution falls to a level indistinguishable from noise (i.e. fully encloses the source). This provides a less biased estimate of the spectrum since it makes no assumption on the source morphology, but it decreases the signalto-noise ratio since some additional background is included compared to an angular cut optimized for best significance. An energy estimate for each event is calculated based on a comparison of the event's impact parameter, zenith angle, offset from the center of the field of view, and the amplitude of the integrated image for each telescope. The energy estimates for all events in the on and off-source regions are put into two histograms, which are then corrected for differing exposure, subtracted, and a flux is calculated for each energy bin by dividing by the observation time and the effective collection area of the telescopes (which is a function of energy, offset from the camera center, zenith angle, and the angle with respect to the Earth's geomagnetic field, as determined from simulations). The resulting fluxes are fit by a power-law of the form

$F(E)=N_{0}\left(\frac{E}{1 \mathrm{TeV}}\right)^{-\Gamma}$

where $\Gamma$ is the photon index and $N_{0}$ is the flux normalization. Muon images are used to correct the energy estimate for changes 
in the optical efficiency of the telescopes over time (due to, e.g. the degradation of the mirrors) (Aharonian et al. 2006b). The systematic error on the flux is conservatively estimated from simulated data to be $20 \%$ while the photon index has a typical systematic error of \pm 0.2 .

To check the robustness of the results presented in this article, the analysis has been repeated using several other background models as well as with a completely separate analysis and calibration chain which used independent simulations and the forward-folded spectrum reconstruction technique described in Piron et al. (2001).

\subsection{Counterpart search}

A search for counterparts to the VHE emission was made by first looking in source catalogs for objects which are of a type known to produce VHE photons, including the ATNF pulsar catalog (Manchester et al. 2005), the Green's supernova remnant catalog (Green 2004), and the High-Mass X-ray binary (HMXB) catalog by Liu et al. (2006). We also checked the Low-Mass Xray binary (LMXB) catalog by Liu et al. (2007), the INTEGRAL source catalog (Bird et al. 2007), and the SIMBAD database. Sky maps for longer-wavelength survey data in the radio and X-ray wavebands, from the Molonglo (Green et al. 1999; Mauch et al. 2003), NRAO VLA (Condon et al. 1998), ROSAT (Voges et al. 2000), ASCA (Tanaka et al. 1994) Galactic plane surveys, were compared with the HESS excess maps. Additionally, pointed observations made by the Chandra and XMM-Newton instruments were checked when available in the respective archives. Unless otherwise noted, ROSAT survey data between 1.0-2.4 keV and ASCA data between 2-10 keV have been used.

To reduce the number of chance coincidences with cataloged sources, some loose selection criteria were applied:

- Based on previous detections in the VHE energy range (e.g. Aharonian et al. 2006c,e), we consider the association of a VHE source with a shell-type SNR plausible only if the VHE emission roughly matches the angular size of the remnant and is not significantly offset.

- Due to the large number of cataloged pulsars in the galactic plane, only those which are energetic enough to power a PWN which could produce VHE emission were considered. A useful quantity for determining the possibility of VHE emission from pulsars is the spin-down flux measured at the solar-system, $\dot{E} / D^{2}$, where $\dot{E}$ is the spin-down luminosity and $D^{2}$ is the distance to the object (both measurable quantities) (Fierro et al. 1995). Defining the conversion efficiency, $\eta$, as the ratio of the integral energy flux of a gamma-ray source over a typical energy range (e.g. $200 \mathrm{GeV}$ to $20 \mathrm{TeV}$ ) to the pulsar spin-down flux at the solar system, we find that for typical spectral characteristics of the sources discussed here, $\dot{E} / D^{2}$ must be well above $10^{33} \mathrm{erg} \mathrm{s}^{-1} \mathrm{kpc}^{-2}$ to produce the observed emission, even assuming $100 \%$ efficiency; for this reason, pulsars with lower spin-down fluxes are not plotted in the figures given later in this paper. In cases where the distance estimate is not known, we assume a distance of $3 \mathrm{kpc}$. We note that efficiencies greater than $100 \%$ are not completely excluded, since the spin-down flux might have been higher in the past and the particle cooling times might be comparable to the pulsar's age (Aharonian et al. 2007d). However, to claim a plausible identification of a VHE source with a pulsar, we require efficiencies of $<10 \%$ and a reasonably small angular distance for the purpose of this study to keep the number of chance coincidences low, unless there
Table 3. Results from an elongated 2-D Gaussian model fit (see Sect. 2.3) to the gamma-ray excess for each source. $\sigma_{1}$ and $\sigma_{2}$ are the intrinsic semi-major and semi-minor axes (in degrees on the sky), with the effect of the point-spread function removed. The errors are statistical. The position angle is measured counter-clockwise in degrees relative to the RA axis.

\begin{tabular}{lccc}
\hline \hline Source & $\sigma_{1}\left(^{\circ}\right)$ & $\sigma_{2}\left(^{\circ}\right)$ & Angle $\left(^{\circ}\right)$ \\
\hline HESS J1427-608 & $0.04 \pm 0.02$ & $0.08 \pm 0.03$ & $80 \pm 17$ \\
HESS J1626-490 & $0.07 \pm 0.02$ & $0.10 \pm 0.05$ & $3 \pm 40$ \\
HESS J1702-420 & $0.30 \pm 0.02$ & $0.15 \pm 0.01$ & $68 \pm 7$ \\
HESS J1708-410 & $0.06 \pm 0.01$ & $0.08 \pm 0.01$ & $-20 \pm 23$ \\
HESS J1731-347 & $0.18 \pm 0.07$ & $0.11 \pm 0.03$ & $-89 \pm 21$ \\
HESS J1841-055 & $0.41 \pm 0.04$ & $0.25 \pm 0.02$ & $39 \pm 6$ \\
HESS J1857+026 & $0.11 \pm 0.08$ & $0.08 \pm 0.03$ & $-3 \pm 49$ \\
HESS J1858+020 & $0.08 \pm 0.02$ & $0.02 \pm 0.04$ & $4 \pm 17$ \\
\hline
\end{tabular}

are other multi-frequency data that would support the association.

- XRBs from which VHE emission is established are HMXBs that either exhibit a jet (e.g. LS 5039, Aharonian et al. 2006f), or where the compact object is a pulsar powering a PWN (e.g. PSR B1259, Aharonian et al. 2005d); all appear variable and point-like in the VHE band. We believe the chance probability of the appearance of an XRB within the $3 \sigma$ contours of an extended HESS source to be reasonably low, therefore we discuss such associations, but for the moment ignore XRBs lying outside the sources. Although the possibility exists that such an object might also power an extended, possibly asymmetric VHE source (e.g. Cheng et al. 2006), this has so far not been observed.

\section{Results}

Results of the size and spectral fits for each source are summarized in Tables 3 and 4, respectively. The spectrum for each source is plotted in Fig. 8. In the following sections, a detailed discussion of each source and related cataloged sources or hotspots within each field of view is given.

\subsection{HESS J1427-608}

HESS J1427-608 (Fig. 1) is located approximately $1^{\circ}$ away from the hard X-ray and GeV gamma-ray source G313.2+0.3 (a strong radio source located in the Kookaburra complex) (Aharonian et al. 2006a), and has a slightly extended morphology consistent with a symmetric Gaussian of radius $\sigma=3^{\prime}$. Its spectrum is fit by a power-law with index $2.2 \pm 0.1_{\text {stat }} \pm 0.2_{\text {sys }}$. Radio and X-ray survey data of the region (overlaid in Fig. 1 from the Molonglo and ROSAT surveys, respectively) show no evidence for significant emission at distances of $0.5^{\circ}$ or closer to the centroid position of HESS J1427-608. There are no nearby pulsars or supernova remnants, and an association of HESS J1427-608 with the unidentified INTEGRAL source IGR J14331-6112 is unlikely due to the large angular distance separating the two sources.

\subsection{HESS J1626-490}

HESS J1626-490, located exactly on the Galactic plane (Fig. 2), is a gamma-ray source with an approximately radiallysymmetric Gaussian morphology (with 5' extent), and a powerlaw energy spectrum with photon index $2.2 \pm 0.1_{\text {stat }} \pm 0.2_{\text {sys }}$. 
Table 4. Summary of spectral parameters for each source from a power-law fit to the spectral data $\left(E=N_{0} E^{-\Gamma}\right)$ over the energy range $E_{\min }-E_{\max }$. The integration radius, $\theta_{\text {int }}$ is chosen to fully enclose each source. Only data with observation positions offset less than $2^{\circ}$ from the source position were included. The errors shown are statistical; the systematic error is conservatively estimated to be $20 \%$ on the flux and \pm 0.2 on the spectral index. Plots of all spectra are given in Fig. 8.

\begin{tabular}{l|cccc|cc|ccc}
\hline \hline Source & $\begin{array}{c}\theta_{\text {int }} \\
\left({ }^{\circ}\right)\end{array}$ & $\begin{array}{c}\text { Time } \\
(\mathrm{hrs})\end{array}$ & $\begin{array}{c}S \\
(\sigma)\end{array}$ & $\begin{array}{c}\text { Excess } \\
(\text { counts })\end{array}$ & $\Gamma$ & \multicolumn{1}{c}{$\begin{array}{c}N_{0} \times 10^{-12} \\
\left(\mathrm{~cm}^{-2} \mathrm{~s}^{-1} \mathrm{TeV}^{-1}\right)\end{array}$} & $\begin{array}{c}E_{\min } \\
(\mathrm{TeV})\end{array}$ & $\begin{array}{c}E_{\max } \\
(\mathrm{TeV})\end{array}$ & $\frac{\chi^{2}}{\text { d.o.f. }}$ \\
\hline HESS J1427-608 & 0.2 & 21 & 5.6 & 165 & $2.16 \pm 0.14$ & $1.3 \pm 0.4$ & 0.97 & 50 & $4.6 / 6$ \\
HESS J1626-490 & 0.5 & 8 & 6.0 & 167 & $2.18 \pm 0.12$ & $4.9 \pm 0.9$ & 0.60 & 50 & $6.4 / 7$ \\
HESS J1702-420 & 0.6 & 7 & 10.6 & 596 & $2.07 \pm 0.08$ & $9.1 \pm 1.1$ & 0.50 & 50 & $11.3 / 7$ \\
HESS J1708-410 & 0.3 & 45 & 10.3 & 542 & $2.46 \pm 0.08$ & $2.7 \pm 0.3$ & 0.50 & 60 & $5.4 / 5$ \\
HESS J1731-347 & 0.6 & 11 & 8.3 & 495 & $2.26 \pm 0.10$ & $6.1 \pm 0.8$ & 0.50 & 80 & $2.8 / 6$ \\
HESS J1841-055 & 0.7 & 10 & 10.7 & 723 & $2.41 \pm 0.08$ & $12.8 \pm 1.3$ & 0.54 & 80 & $10.4 / 6$ \\
HESS J1857+026 & 0.46 & 15 & 10.2 & 425 & $2.39 \pm 0.08$ & $6.1 \pm 0.7$ & 0.60 & 80 & $5.9 / 6$ \\
HESS J1858+020 & 0.15 & 23 & 7.4 & 116 & $2.17 \pm 0.12$ & $0.6 \pm 0.1$ & 0.50 & 80 & $4.9 / 6$ \\
\hline
\end{tabular}
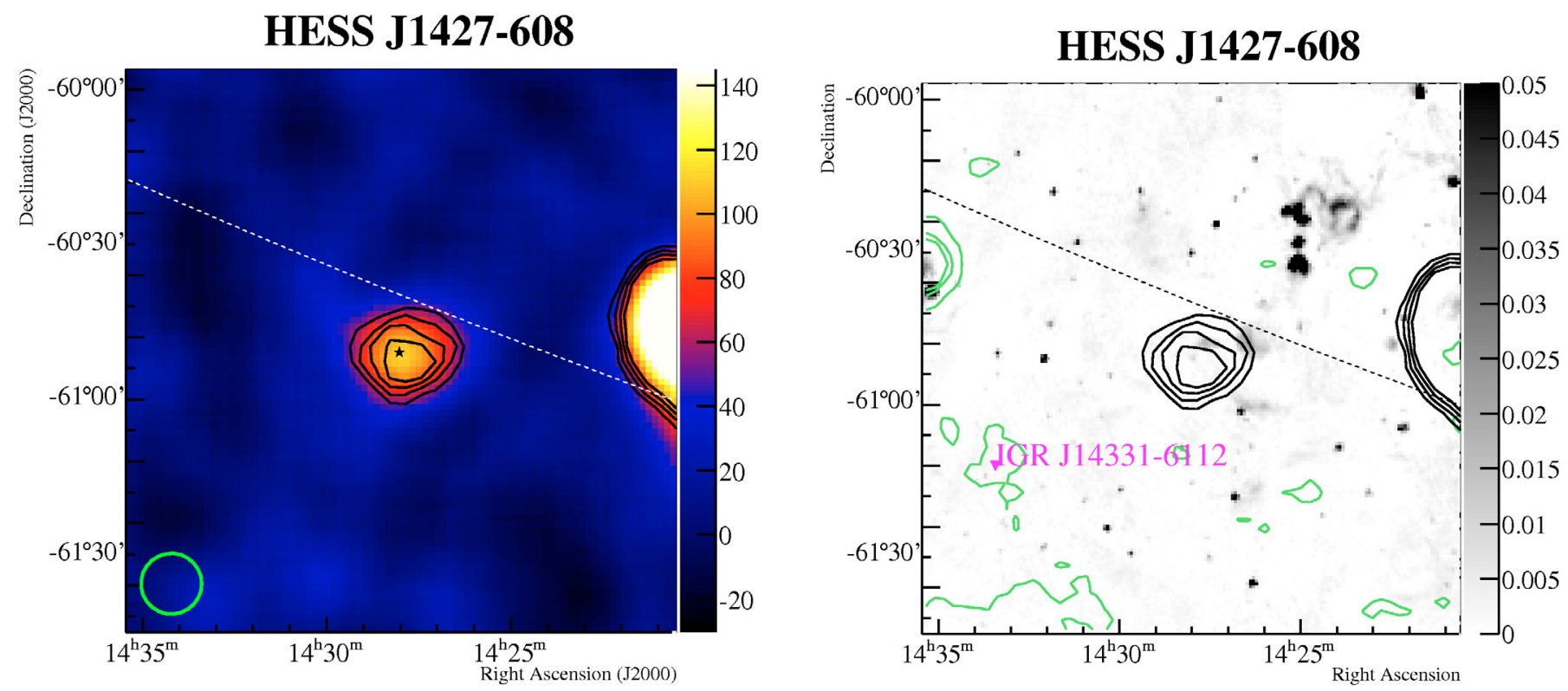

Fig. 1. Left: a VHE gamma-ray image of HESS J1427-608 (center position marked with a star). The image is of gamma-ray excess counts smoothed with a Gaussian filter with standard deviation $0.1^{\circ}$ (referred to as the smoothing radius hereafter). The smoothing radius is chosen according to event statistics and therefore differs from source to source and is shown as a green circle in the lower left-hand corner. The color scale of the image is set such that the blue/red transition occurs at approximately the $3 \sigma$ (pre-trials) significance level. Overlaid on the image are the significance contours starting at $4 \sigma$ in $1 \sigma$ steps. The Galactic plane is marked with a dashed line. The gamma-ray excess at the right of the image is the hard X-ray source known as the Kookaburra/Rabbit, which is discussed in Aharonian et al. (2006a). Right: the HESS significance contours (black) overlaid on a radio image (Green et al. 1999) (grey-scale, in Jy/beam). The green contours are from a ROSAT hard-band X-ray image (Voges et al. 2000) which has been adaptively smoothed with the FTOOLS fadapt algorithm to accentuate diffuse emission (Blackburn 1995). Also plotted are ATNF pulsars with $\dot{E} / D^{2} \geq 10^{33} \mathrm{erg} \mathrm{s}^{-1} \mathrm{kpc}^{2}$, SNRs from Green's catalog, HMXBs and LMXBs from the catalogs of Liu et al, and INTEGRAL sources (see Sect. 2.4 for references). In this case, only the INTEGRAL source IGR J14331-6112 lies within the field of view.

There is a slight extension toward increasing right ascension which is only marginally significant, but may be an indication of a second VHE source. Within the gamma-ray emission region, there exists some weak radio emission, along with the unidentified X-ray source 1RXS J162504-490918, which lies approximately $10^{\prime}$ from the centroid position and is a possible $\mathrm{X}$-ray counterpart. This X-ray source, marked with an " $\mathrm{X}$ " in the figure, has an extent of $13^{\prime \prime}$ and an absorption-corrected flux between $0.1-2.0 \mathrm{keV}$ of $1.7 \times 10^{-13} \mathrm{erg} \mathrm{cm}^{-2} \mathrm{~s}^{-1}$, assuming a photon index of 2.0 (Voges et al. 2000; Mukai 1993). The shelltype supernova remnant G335.2+00.1 (MSH 16-44) (Whiteoak \& Green 1996) lies just outside the significant emission region of HESS J1626-490, as does the LMXB 4U 1624-490 (Smale et al. 2000), and the HMXB IGR 16283-4838 (Bird et al. 2007), which are not considered plausible candidates due to their offsets.

\subsection{HESS J1702-420}

First discovered by HESS at an approximately $6 \sigma$ significance level (Aharonian et al. 2006d), HESS J1702-420 (Fig. 3) is now seen with increased observation time at a significance level of $13 \sigma$. Its spectrum is characterized by a power-law with in$\operatorname{dex} 2.1 \pm 0.1_{\text {stat }} \pm 0.2_{\text {sys }}$, slightly harder than the previously reported value of $2.3 \pm 0.15_{\text {stat }} \pm 0.2_{\text {sys }}$, which was derived from a smaller integration radius, less statistics, and over a smaller energy range. The results, including the source location, are consistent within the errors. The emission "tail" extending to positive galactic longitude and latitude is statistically significant, giving the source an elongated morphology (see Table 3). The nearby pulsar PSR J1702-4128 (to the north of the VHE emission region, Fig. 3) lies at the edge of the gamma-ray emission, and with $\dot{E} / D^{2}=1.3 \times 10^{34} \mathrm{erg} \mathrm{s}^{-1} \mathrm{kpc}^{-2}$, it provides enough 

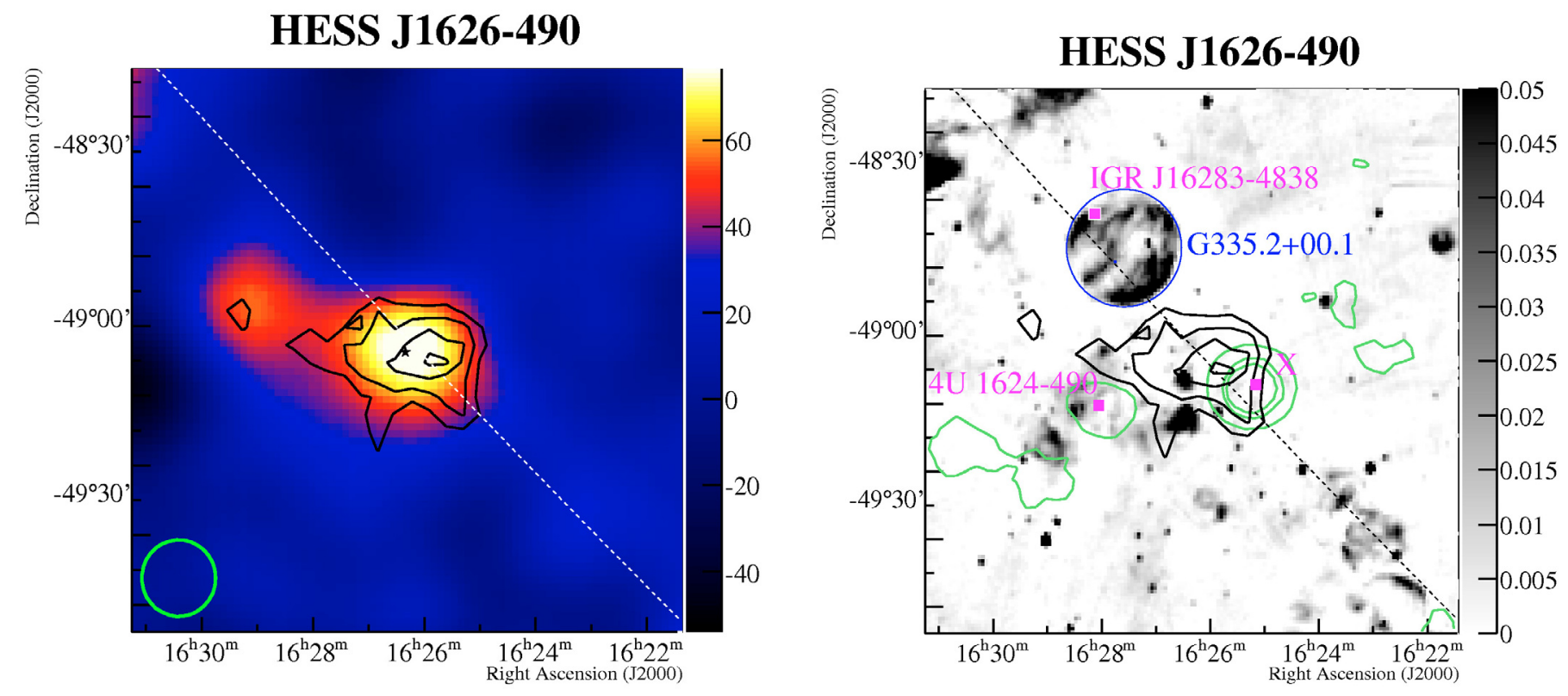

Fig. 2. Left: a VHE gamma-ray image of HESS J1626-490 plotted as in Fig. 1, with a smoothing radius of $0.1^{\circ}$. Right: the HESS significance (black) and adaptively smoothed ROSAT X-ray contours (green), overlaid on the Molonglo radio image (grey-scale). Also plotted is the SNR G335.2+00.1 (circle marking extent), the HMXB IGR 16283-4838, the LMXB 4U 1624-490, and the unidentified X-ray source 1RXS J162504490918 (labeled $X$ ).
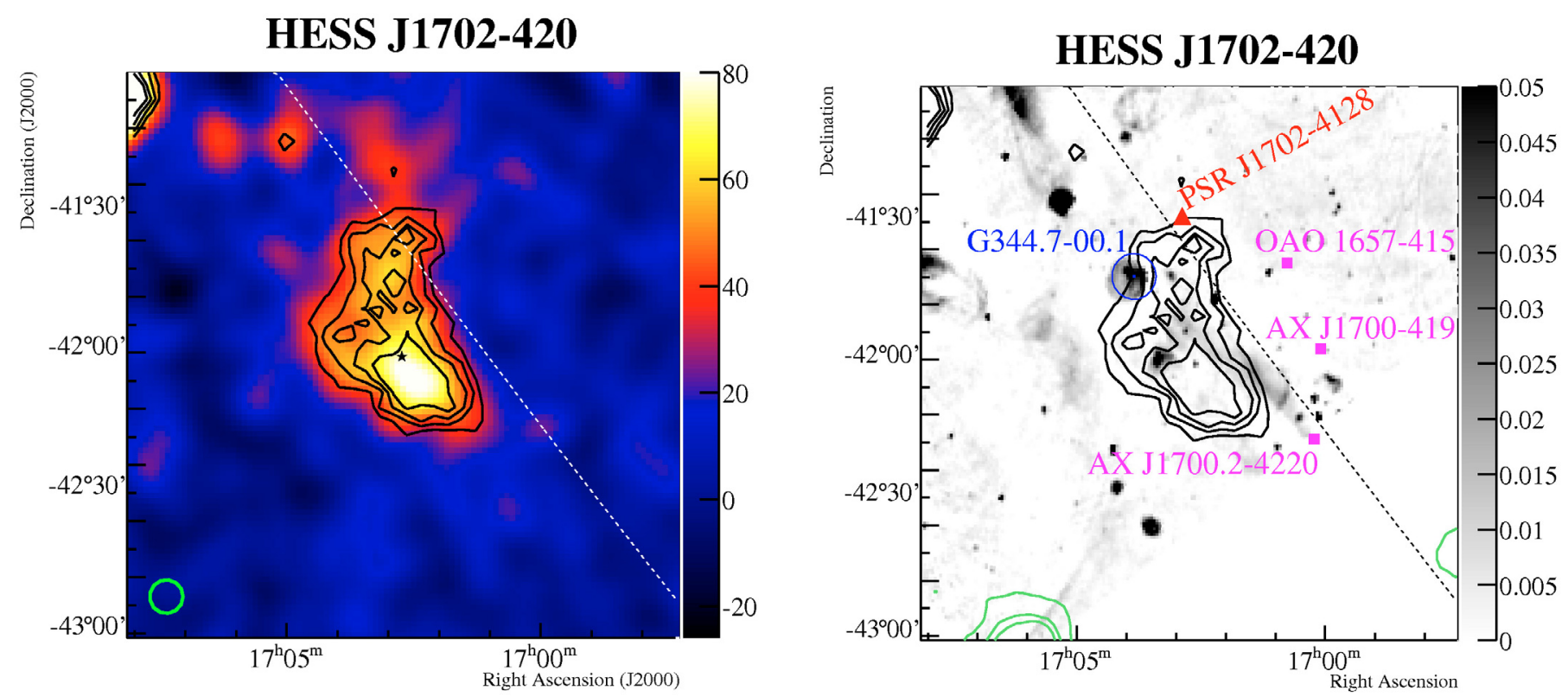

Fig. 3. Left: a VHE gamma-ray image of HESS J1702-420, plotted as in Fig. 1, with a smoothing radius of $0.06^{\circ}$. Right: the HESS significance (black) and adaptively smoothed ROSAT X-ray contours (green), overlaid on the Molonglo radio image (grey-scale). Also plotted are the positions of the SNR G344.7-00.1 (circle), three HMXBs (magenta squares), and the high spin-down flux pulsar PSR J1702-4128 (red triangle).

spin-down energy loss to produce the observed emission (assuming a rather high conversion efficiency of $\sim 70 \%$ if the present distance estimate of $5 \mathrm{kpc}$ is correct) and may be a counterpart if it powers an extremely asymmetric pulsar wind nebula. The nearby shell-type supernova remnant G344.7-00.1 (seen in the radio image) is also detected by ASCA in the $2-10 \mathrm{keV}$ X-ray energy band (Sugizaki et al. 2001), however is an unlikely counterpart due to its small angular size and distance from the peak of the emission region. Three $\mathrm{X}$-ray binaries are also located nearby the source (see the figure), but are outside the significant emission region.

\subsection{HESS J1708-410}

HESS J1708-410 (Fig. 4), situated between the supernova remnant RXJ 1713.7-3946 (Aharonian et al. 2006e) and HESS J1702-420, was first reported at a significance level of approximately $7 \sigma$ (Aharonian et al. 2006d). With additional observations of the region (mostly from the edge of pointed observations centered on RXJ1713.7-3946), the data set now has a statistical significance of $11 \sigma$. The spectrum is fit by a power-law with index $2.5 \pm 0.1_{\text {stat }} \pm 0.2_{\text {sys }}$, which is slightly softer than the previously published result of $2.3 \pm 0.1_{\text {stat }} \pm 0.2_{\text {sys }}$ made with 

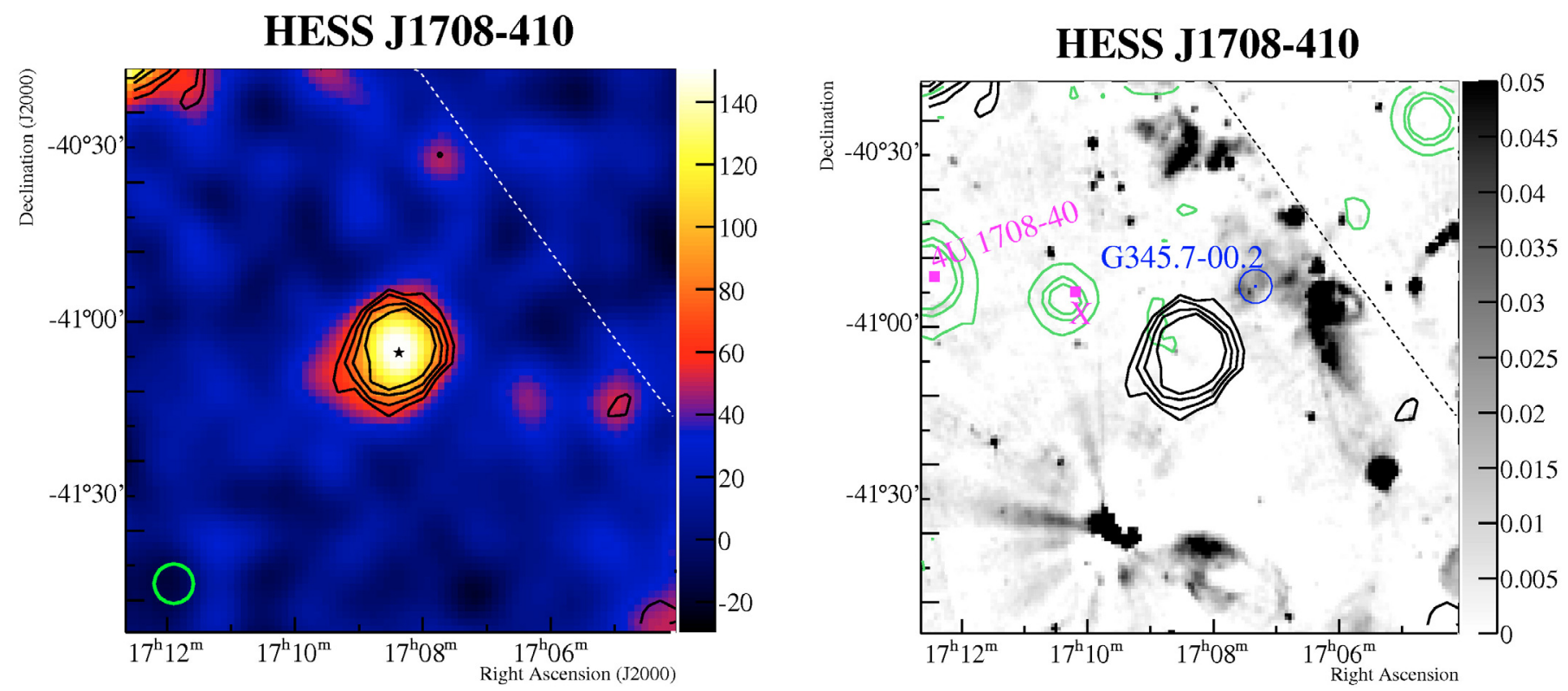

Fig. 4. Left: a VHE gamma-ray image of HESS J1708-410 plotted as in Fig. 1, with a smoothing radius of $0.06^{\circ}$. The slight excess seen on the lower-right corner of the image is HESS J1702-420 (see previous section), while the excess seen at the upper-left corner is part of RXJ 1713.73946 (Aharonian et al. 2006e). Right: the HESS significance (black) and adaptively smoothed ROSAT X-ray contours (green), overlaid on the Molonglo radio image (grey-scale). Also plotted are the positions of the SNR G345.7-00.2, the ROSAT source 1RXS J171011.5-405356 (labeled "X"), and the LMXB 4U 1708-40 (square).

lower statistics, a smaller integration radius, and over a smaller energy range (Aharonian et al. 2006d), though is within errors. The compact morphology of HESS J1708-410 is consistent with a slightly elongated Gaussian of approximately $0.08^{\circ}$ extent, with no significant emission beyond $0.3^{\circ}$, ruling out SNR G345.7-00.2 or nearby radio hot-spots as obvious counterpart candidates. Although several ROSAT hard-band X-ray hot spots exist in the field-of-view (e.g. the XRB 4U 1708-40 or 1RXS J171011.5-405356, see figure), the closest is $0.2^{\circ}$ away and is not obviously connected with the gamma-ray emission. There is an XMM-Newton exposure centered on G345.7-00.2, in which no significant emission is seen near the VHE position. Additionally, an ASCA exposure of the region reveals only a single point-like source located over a degree from the HESS source.

\subsection{HESS J1731-347}

HESS J1731-347 (Fig. 5) is detected at an $\sim 8 \sigma$ level, exhibiting a power-law spectral index of $2.3 \pm 0.1_{\text {stat }} \pm 0.2_{\text {sys. }}$. The source has a significant tail which extends westward, giving it a nonGaussian morphology, possibly indicating the presence of more than one or an extended non-uniform source. A slice in the uncorrelated excess event map along the axis of the emission does not show a conclusive separation between the two "peaks", and a spectral analysis of each gives the same photon index within systematic errors. For this reason, the emission is treated here as a single source.

A bright X-ray point source (1RXS J173030.3-343219, labeled as " $X$ " in the figure) is seen in the ROSAT data, approximately 0.4 degrees in the direction of the Galactic Plane from the centroid position, and has an absorption-corrected flux in the $0.1-2.4 \mathrm{keV}$ range of approximately $2.0 \times 10^{-11} \mathrm{erg} \mathrm{cm}^{-2} \mathrm{~s}^{-1}$ (Voges et al. 1999; Mukai 1993), assuming a spectral index of 2.0. This source is identified with the cataclysmic variable (CV) star HD 158394, and is not expected to produce VHE emission. However, around the brightest part of the TeV emission, there is some unidentified nebular X-ray emission that partially matches the morphology of the HESS source, and may well be the X-ray counterpart. This diffuse X-ray emission includes the extended ROSAT source 1RXS J173251.1-344728 (labeled $X_{1}$ in the figure), which has an extension of $2^{\prime}$ and Xray flux of $(7 \pm 1) \times 10^{-12} \mathrm{erg} \mathrm{cm}^{-2} \mathrm{~s}^{-1}$, and a nearly coincident point-like radio source labeled 353.464-0.69 in the VLA survey data (Zoonematkermani et al. 1990); their association with the VHE emission is unclear. The strong point-like radio source 173028-344144 (Condon et al. 1998), labeled $R$ in the figure, also lies to the right of the peak of the VHE emission. The X-ray emission about a degree away to the north in the figure comes from the LMXB GX 354-0, however due to its distance from HESS J1731-347 and since these objects are not known to produce extended gamma-ray emission, it is an unlikely counterpart candidate. No known high spin-down flux pulsars lie within the emission region.

\subsection{HESS J1841-055}

HESS J1841-055 (Fig. 6) exhibits a highly extended, possibly two or three-peaked , morphology; however, the "dip" between the peaks along the major axis is not statistically significant $(<1.5 \sigma)$. The source has a spectrum that is fit by a power law with index $2.4 \pm 0.1_{\text {stat }} \pm 0.2_{\text {sys }}$. An association with either pulsar PSR J1841-0524 $\left(\dot{E} / D^{2}=4.4 \times 10^{33} \mathrm{erg} \mathrm{s}^{-1} \mathrm{kpc}^{-2}\right)$ or PSR J1838-0549 $\left(\dot{E} / D^{2}=4.7 \times 10^{33} \mathrm{erg} \mathrm{s}^{-1} \mathrm{kpc}^{-2}\right)$, is not ruled out, however taken separately, each would require approximately $200 \%$ efficiency to explain the VHE emission. This is not completely implausible if both pulsars contribute together or if either had a much higher spin-down luminosity in the past (PSR J18380549 is estimated to have a relatively old characteristic age of 112 kyr, while PSR J1841-0524 is about $30 \mathrm{kyr}$ old Manchester et al. 2005). PSR J1837-0604 ( $\left.\dot{E} / D^{2}=5.2 \times 10^{34} \mathrm{erg} \mathrm{s}^{-1} \mathrm{kpc}^{-2}\right)$ has a high enough spin-down flux to be a counterpart candidate, 

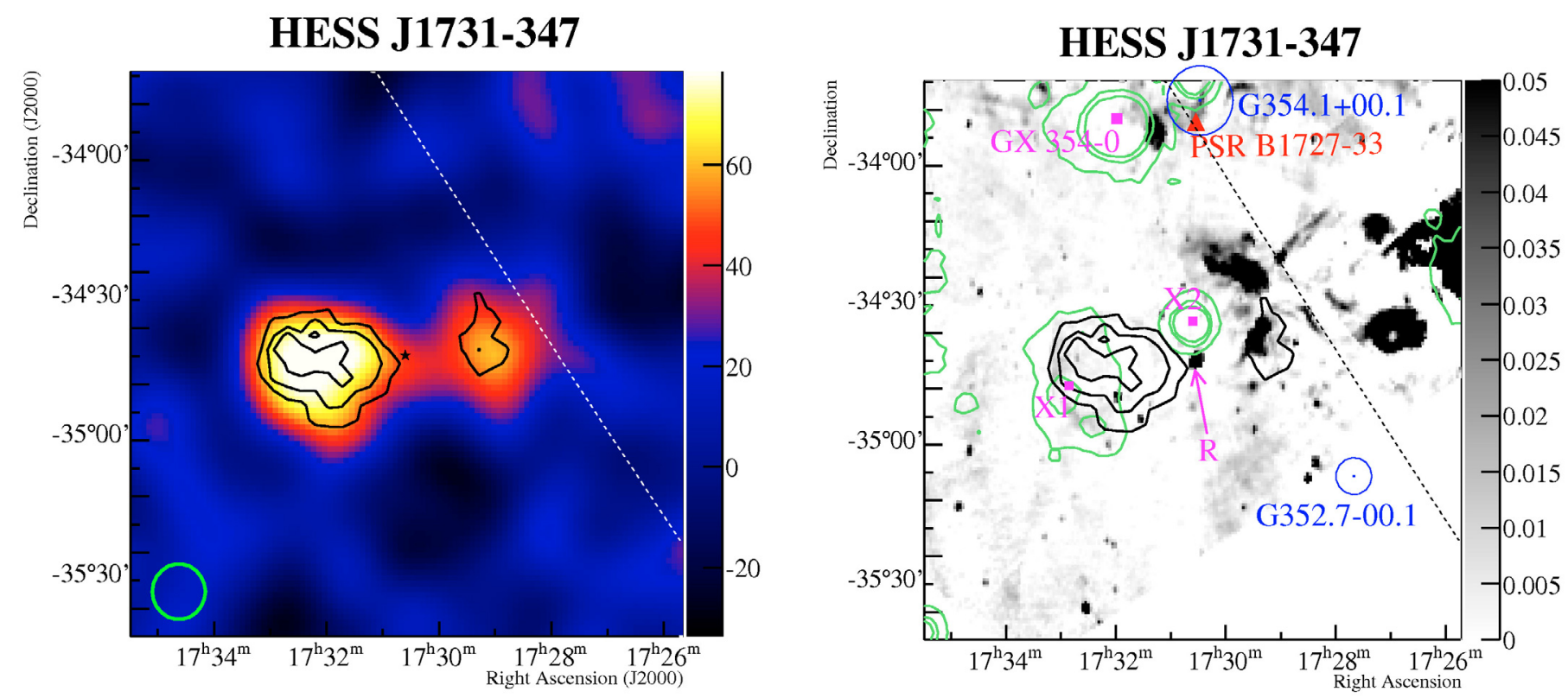

Fig. 5. Left: a VHE gamma-ray image of HESS J1731-347 plotted as in Fig. 1, with a smoothing radius of $0.1^{\circ}$. Right: the HESS significance (black) and adaptively smoothed ROSAT X-ray contours (green), overlaid on the Molonglo radio image (grey-scale). Also shown are the positions of a high spin-down flux pulsar (filled triangle), the low-mass X-ray binary GX 354-0, and cataloged supernova remnants (blue circles marking

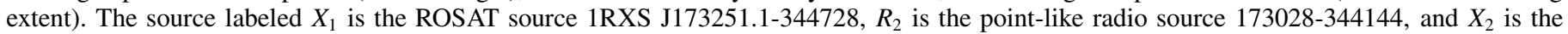
ROSAT point-source 1RXS J173030.3-343219.
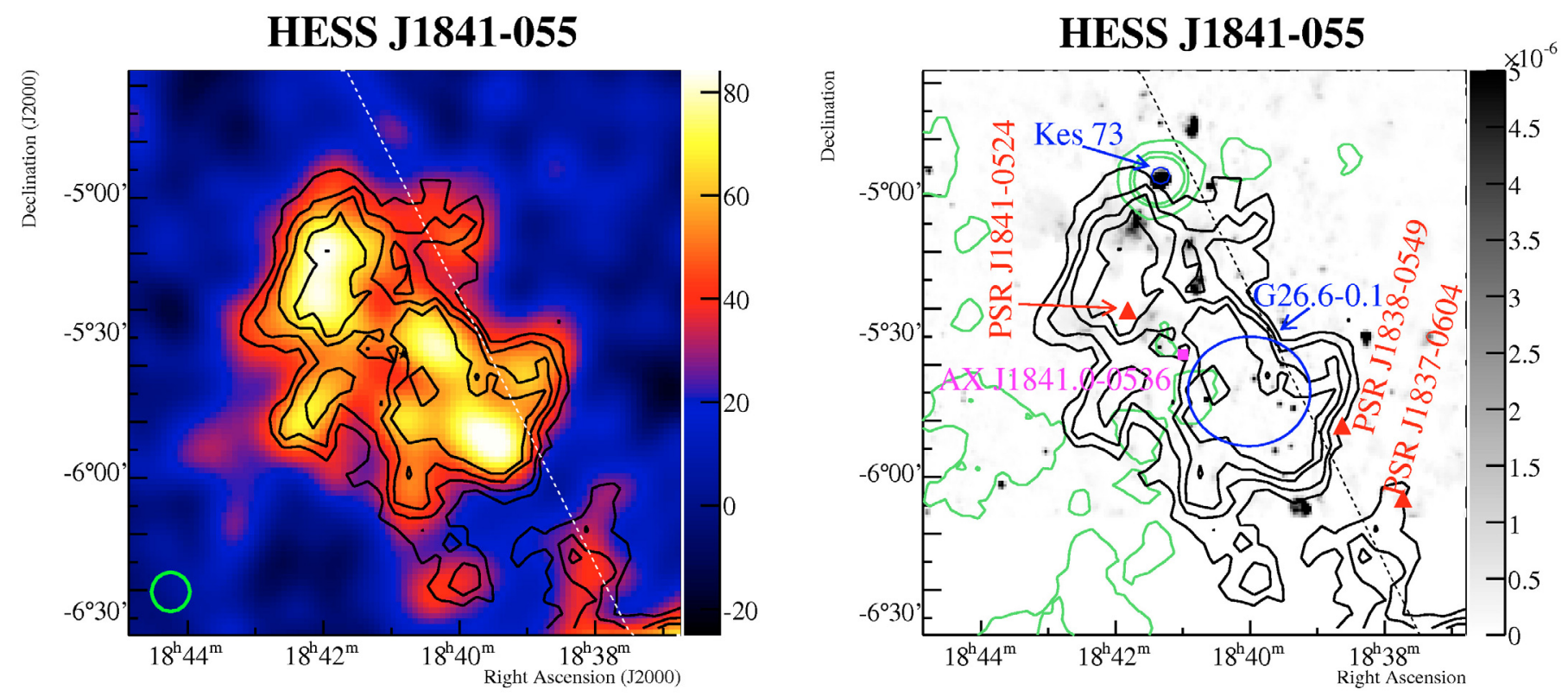

Fig. 6. Left: a VHE gamma-ray image of HESS J1841-055, plotted as in Fig. 1, with a smoothing radius of $0.07^{\circ}$. Right: the HESS significance (black) and adaptively smoothed ROSAT X-ray contours (green), overlaid on the NVSS radio image (grey-scale). Also shown are the positions of known high spin-down flux pulsars (filled triangles), the SNR Kes 73 (circle), the X-ray candidate SNR G26.6-0.1 (blue circle), and the HMXB AXJ 1841.0-0536. The Ginga source GS 1839-06 is compatible with the location of AXJ 1841.0-0536.

however it is well outside the emission region. There are no cataloged PWN at longer wavelengths identified with any of the three pulsars (e.g. Gotthelf 2004). The SNR G027.4+00.0 (also known as Kes 73), which is visible in both X-ray and radio wave bands, lies at the edge of the emission, though does not appear related due to its small angular size. Additionally, the high-mass X-ray binary J1839-06 also lies near the edge of the significant TeV excess.

ASCA observations of the Scutum arm region reveal a point-like source, AX J1841.0-0536, near the center of the VHE emission, which based on its X-ray light curve and optical emission is suggested to be a Be/X-ray binary pulsar (Bamba et al. 2001) with a flux in the 6-20 keV energy range of $1.1 \times 10^{-10} \mathrm{erg} \mathrm{cm}^{-2} \mathrm{~s}^{-1}$ and photon index of $2.2 \pm 0.3$ (Filippova et al. 2005). A Chandra observation of this object confirms the identification, with a flux in the $0.5-10 \mathrm{keV}$ energy range of $4.2 \times 10^{-12} \mathrm{erg} \mathrm{cm}^{-2} \mathrm{~s}^{-1}$ (Halpern et al. 2004). Given its pointlike extent, AX J1841.0-0536 is not large enough to explain the entire HESS source, however it may well be responsible for a component of the emission. 
HESS J1857+026, HESS J1858+020

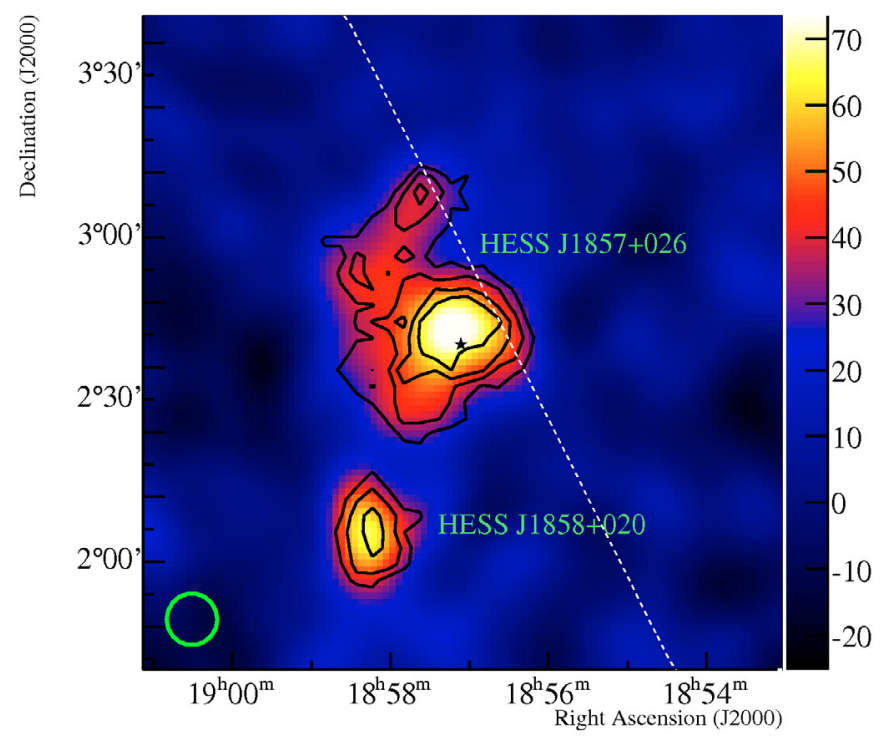

HESS J1857+026, HESS J1858+020

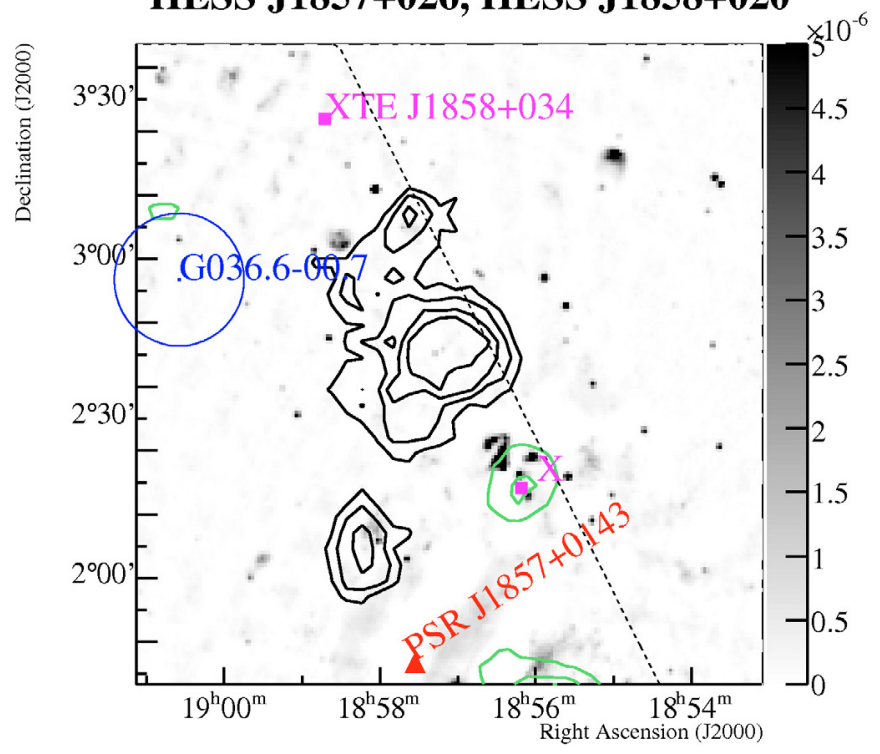

Fig. 7. Left: a VHE gamma-ray image of HESS J1857+026 and HESS J1858+020, plotted as in Fig. 1, with a smoothing radius of $0.08^{\circ}$. Right: the HESS significance (black) and adaptively smoothed ROSAT X-ray contours (green), overlaid on an NVSS radio image (grey-scale). Also shown are the positions of a known high spin-down flux pulsar (filled triangle), the SNR G036.6-00.7 (circle), the ROSAT point-source 1RXS J185609.2+021744 (labeled X), and the HMXB XTE J1858+034 (square).

Also within the VHE emission region lies the diffuse source G26.6-0.1, which was detected in the ASCA Galactic Plane Survey and is postulated based on its spectrum to be a candidate supernova remnant (Bamba et al. 2003), and is also coincident with an H II region (Lockman 1989). With its angular size of $8.3^{\prime}(F W H M)$, small distance (approximately $1.3 \mathrm{kpc}$ ), and nonthermal spectrum, this object also may also contribute to a component of the VHE emission. Additionally, the nearby source AX J18406-0539 is possibly an XRB at a distance of $1.1 \mathrm{kpc}$ (Masetti et al. 2006), though given positional errors, may well be the same source as AX J1841.0-0536 (Negueruela \& Schurch 2007).

\subsection{HESS J1857+026}

HESS J1857+026 (Fig. 7) is an approximately radiallysymmetric extended VHE gamma-ray source located on the Galactic Plane. The source is detected by HESS at a $9 \sigma$ significance level at energies above $300 \mathrm{GeV}$ and has a differential spectral index of $2.4 \pm 0.1_{\text {stat }} \pm 0.2_{\text {sys. }}$. The slight extension of the source seen toward the north is significant $(\sim 5 \sigma)$ and may indicate a more extended morphology or the presence of a weaker nearby source, though more observation time will be needed to make a conclusive statement.

This source lies approximately $0.7^{\circ}$ from HESS J1858+020 (see Sect. 3.8), which is most probably a separate source since no significant emission connects the two. An association with the supernova remnant G036.6-00.7, which lies over a degree from the centroid position, is unlikely. Though an ASCA observation exists which is roughly centered on the source position, no excess was seen, implying a $95 \%$ absorbed flux upper-limit of $1.2 \times 10^{-12} \mathrm{erg} \mathrm{cm}^{-2} \mathrm{~s}^{-1}$ between $2-10 \mathrm{keV}$. The X-ray source seen about a quarter of a degree from the centroid position is the point-source 1RXS J185609.2+021744 (labeled $X$ in the figure, and coincident with the ASCA source AXJ 185608+0218), which has a flux in the $0.1-2.4 \mathrm{keV}$ range of $(0.32 \pm 0.06) \times$ $10^{-12} \mathrm{erg} \mathrm{cm}^{-2} \mathrm{~s}^{-1}$, assuming a photon index of 2.0; its distance from the emission region makes it an unlikely counterpart candidate, however.

\subsection{HESS J1858+020}

The weak gamma-ray source HESS J1858+020 (shown also in Fig. 7) lies close to HESS J1857+026; however, there is no significant emission connecting them, suggesting that they are distinct objects. It is detected at a significance level of $7 \sigma$ with a differential spectral index of $2.2 \pm 0.1_{\text {stat }} \pm 0.2_{\text {sys. }}$. Though nearly point-like, its morphology shows a slight extension of $\sim 5^{\prime}$ along its major axis. PSR J1857+0143 $\left(\dot{E} / D^{2}=1.7 \times\right.$ $10^{34} \mathrm{erg} \mathrm{s}^{-1} \mathrm{kpc}^{-2}$ ) is powerful enough to explain the source, but is significantly offset.

\section{Summary}

The eight VHE gamma-ray sources discussed here are all extended objects with angular sizes ranging from approximately 3 to 18 arcmin, lying close to the Galactic plane (suggesting they are located within the Galaxy). In each case, the spectrum of the sources in the $\mathrm{TeV}$ energy range can be characterized as a power-law with a differential spectral index in the range 2.1 to 2.5. The general characteristics of these sources - spectra, size, and position-are similar to previously identified galactic VHE sources (e.g. PWNe), however since these sources have so far no clear counterpart in lower-energy wavebands, further multiwavelength study is required to understand the emission mechanisms powering them, and therefore follow-up observations with higher-sensitivity X-ray and GeV gamma-ray telescopes will be beneficial. Since most VHE sources are predicted to emit X-ray and radio emission, a non-detection of longer-wavelength emission with current-generation experiments for some of these objects may be an indication that a new VHE source class exists (as suggested by Aharonian et al. 2005b), and may provide new insight into high-energy processes within our Galaxy. 

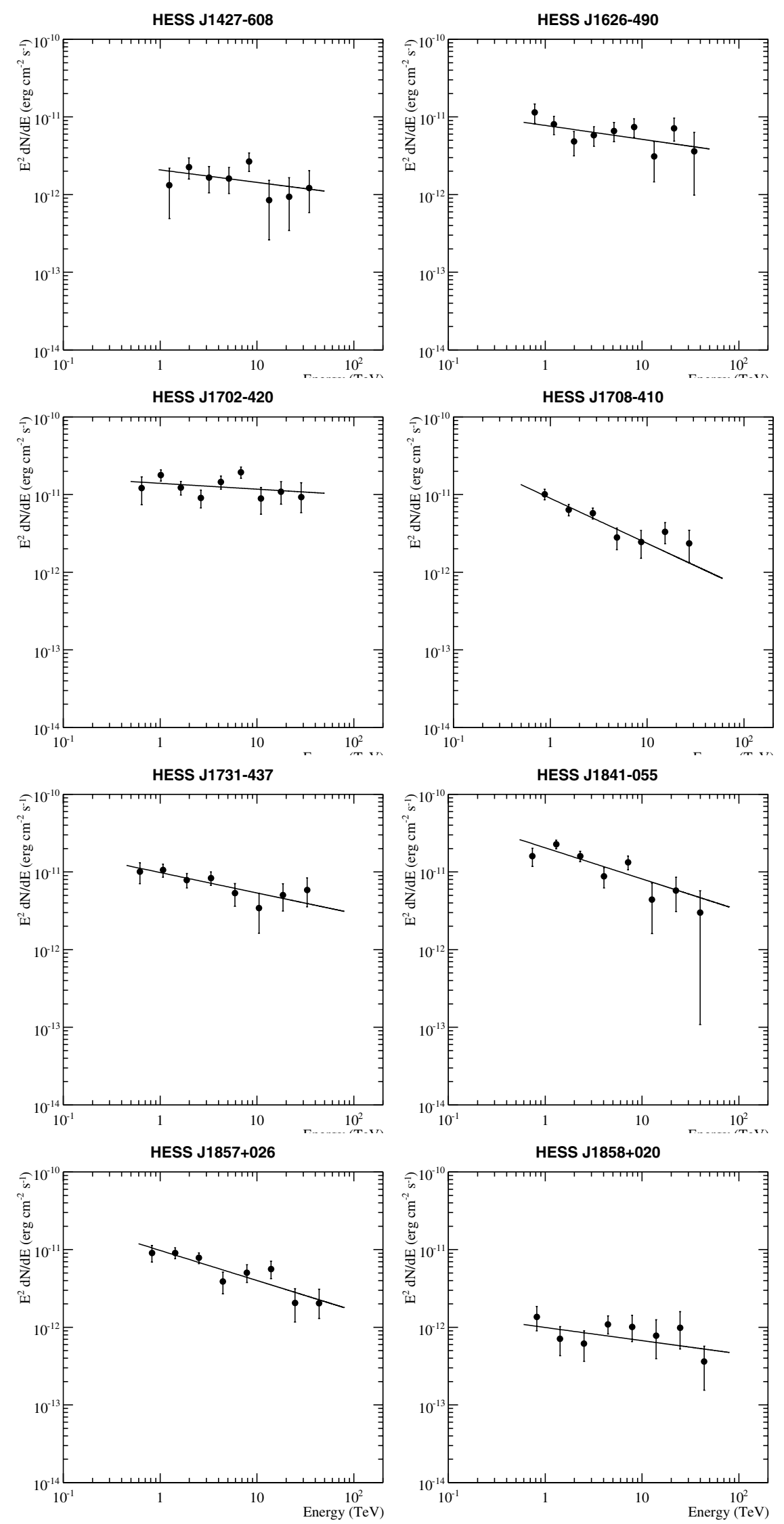

Fig. 8. Spectra for each unidentified source, with power-law fits. See Table 4 for detailed fit information.

Acknowledgements. The support of the Namibian authorities and of the University of Namibia in facilitating the construction and operation of HESS is gratefully acknowledged, as is the support by the German Ministry for Education and Research (BMBF), the Max Planck Society, the French Ministry for
Research, the CNRS-IN2P3 and the Astroparticle Interdisciplinary Programme of the CNRS, the UK Science and Technology Facilities Council (STFC), the IPNP of the Charles University, the Polish Ministry of Science and Higher Education, the South African Department of Science and Technology and 
National Research Foundation, and by the University of Namibia. We appreciate the excellent work of the technical support staff in Berlin, Durham, Hamburg, Heidelberg, Palaiseau, Paris, Saclay, and in Namibia in the construction and operation of the equipment.

This research has made use of the SIMBAD database, operated at CDS, Strasbourg, France and the ROSAT Data Archive of the Max-Planck-Institut für extraterrestrische Physik (MPE) at Garching, Germany.

\section{References}

Aharonian, F., Atoyan, A. M., \& Kifune, T. 1997, MNRAS, 291, 162 Aharonian, F., Akhperjanian, A., Beilicke, M., et al. 2002, A\&A, 393, L37 Aharonian, F., Akhperjanian, A. G., Aye, K.-M., et al. 2004, Astropart. Phys., 22, 109

Aharonian, F., Akhperjanian, A., Beilicke, M., et al. 2005a, A\&A, 431, 197

Aharonian, F., Akhperjanian, A. G., Aye, K.-M., et al. 2005b, Science, 307, 1938

Aharonian, F., Akhperjanian, A. G., Aye, K.-M., et al. 2005c, A\&A, 439, 1013

Aharonian, F., Akhperjanian, A. G., Aye, K.-M., et al. 2005d, A\&A, 442, 1

Aharonian, F., Akhperjanian, A. G., Aye, K.-M., et al. 2006a, in preperation

Aharonian, F., Akhperjanian, A. G., Bazer-Bachi, A. R., et al. 2006b, A\&A, 457, 899

Aharonian, F., Akhperjanian, A. G., Bazer-Bachi, A. R., et al. 2006c, A\&A, 448, L43

Aharonian, F., Akhperjanian, A. G., Bazer-Bachi, A. R., et al. 2006d, ApJ, 636, 777

Aharonian, F., Akhperjanian, A. G., Bazer-Bachi, A. R., et al. 2006e, A\&A, 449, 223

Aharonian, F., Akhperjanian, A. G., Bazer-Bachi, A. R., et al. 2006f, A\&A, 460, 743

Aharonian, F., Akhperjanian, A. G., Bazer-Bachi, A. R., et al. 2007a, A\&A, 469, L1

Aharonian, F., Akhperjanian, A. G., Bazer-Bachi, A. R., et al. 2007b, ArXiv e-prints, 705

Aharonian, F., Akhperjanian, A. G., Bazer-Bachi, A. R., et al. 2007c, A\&A, 467, 1075

Aharonian, F., Akhperjanian, A. G., Bazer-Bachi, A. R., et al. 2007d, in preparation

Atoyan, A., Buckley, J., \& Krawczynski, H. 2006, ApJ, 642, L153

Bamba, A., Ueno, M., Koyama, K., \& Yamauchi, S. 2003, ApJ, 589, 253

Bamba, A., Yokogawa, J., Ueno, M., Koyama, K., \& Yamauchi, S. 2001, PASJ, 53, 1179

Berge, D., Funk, S., \& Hinton, J. 2007, A\&A, 466, 1219

Bernlöhr, K., Carrol, O., Cornils, R., et al. 2003, Astropart. Phys., 20, 111

Bird, A. J., Malizia, A., Bazzano, A., et al. 2007, ApJS, 170, 175

Blackburn, J. K. 1995, in Astronomical Data Analysis Software and Systems IV, ed. R. A. Shaw, H. E. Payne, \& J. J. E. Hayes, ASP Conf. Ser., 77, 367

Cheng, K. S., Taam, R. E., \& Wang, W. 2006, ApJ, 641, 427

Condon, J. J., Cotton, W. D., Greisen, E. W., et al. 1998, AJ, 115, 1693

Daum, A., Hermann, G., Hess, M., et al. 1997, Astropart. Phys., 8, 1

Fierro, J. M., Arzoumanian, Z., Bailes, M., et al. 1995, ApJ, 447, 807

Filippova, E. V., Tsygankov, S. S., Lutovinov, A. A., \& Sunyaev, R. A. 2005, Astron. Lett., 31, 729

Gotthelf, E. V. 2004, in Young Neutron Stars and Their Environments, ed. F. Camilo \& B. M. Gaensler, IAU Symp., 218, 225

Green, D. A. 2004, Bull. Astron. Soc. India, 32, 335

Green, A. J., Cram, L. E., Large, M. I., \& Ye, T. 1999, ApJS, 122, 207

Halpern, J. P., Gotthelf, E. V., Helfand, D. J., Gezari, S., \& Wegner, G. A. 2004, The Astronomer's Telegram, 289, 1

Hillas, A. M. 1996, Space Sci. Rev., 75, 17

Li, T.-P. \& Ma, Y.-Q. 1983, ApJ, 272, 317

Liu, Q. Z., van Paradijs, J., \& van den Heuvel, E. P. J. 2006, A\&A, 455, 1165

Liu, Q. Z., van Paradijs, J., \& van den Heuvel, E. P. J. 2007, A\&A, 469, 807

Lockman, F. J. 1989, ApJS, 71, 469

Manchester, R. N., Hobbs, G. B., Teoh, A., \& Hobbs, M. 2005, AJ, 129, 1993

Masetti, N., Mason, E., Bassani, L., et al. 2006, A\&A, 448, 547

Mauch, T., Murphy, T., Buttery, H. J., et al. 2003, MNRAS, 342, 1117

Mukai, K. 1993, Legacy, 3, 21

Negueruela, I., \& Schurch, M. P. E. 2007, A\&A, 461, 631

Piron, F., Djannati-Atai, A., Punch, M., et al. 2001, A\&A, 374, 895

Smale, A. P., Zhang, W., \& Hertz, P. 2000, in Rossi2000: Astrophysics with the

Rossi X-ray Timing Explorer, March 22-24, 2000 at NASA's Goddard Space

Flight Center, Greenbelt, MD USA, meeting abstract, ed. T. E. Strohmayer

Sugizaki, M., Mitsuda, K., Kaneda, H., et al. 2001, ApJS, 134, 77

Tanaka, Y., Inoue, H., \& Holt, S. S. 1994, PASJ, 46, L37
Voges, W., Aschenbach, B., Boller, T., et al. 1999, A\&A, 349, 389

Voges, W., Aschenbach, B., Boller, T., et al. 2000, IAU Circ., 7432, 3

Weekes, T. C. 1996, Space Sci. Rev., 75, 1

Whiteoak, J. B. Z., \& Green, A. J. 1996, A\&AS, 118, 329

Zoonematkermani, S., Helfand, D. J., Becker, R. H., White, R. L., \& Perley, R. A. 1990, ApJS, 74, 181

${ }^{1}$ Max-Planck-Institut für Kernphysik, PO Box 103980, 69029 Heidelberg, Germany

e-mail: Karl.Kosack@mpi-hd.mpg.de

2 Yerevan Physics Institute, 2 Alikhanian Brothers St., 375036

Yerevan, Armenia

${ }_{3}$ Centre d'Etude Spatiale des Rayonnements, CNRS/UPS, 9 av. du Colonel Roche, BP 4346, 31029 Toulouse Cedex 4, France

${ }^{4}$ Universität Hamburg, Institut für Experimentalphysik, Luruper Chaussee 149, 22761 Hamburg, Germany

5 Institut für Physik, Humboldt-Universität zu Berlin, Newtonstr. 15, 12489 Berlin, Germany

6 LUTH, Observatoire de Paris, CNRS, Université Paris Diderot, 5 Place Jules Janssen, 92190 Meudon, France

7 DAPNIA/DSM/CEA, CE Saclay, 91191 Gif-sur-Yvette Cedex, France

${ }^{8}$ University of Durham, Department of Physics, South Road, Durham DH1 3LE, UK

${ }_{9}$ Unit for Space Physics, North-West University, Potchefstroom 2520, South Africa

${ }_{10}$ Laboratoire Leprince-Ringuet, École Polytechnique, CNRS/IN2P3, 91128 Palaiseau, France

${ }_{11}$ Laboratoire d'Annecy-le-Vieux de Physique des Particules, CNRS/IN2P3, 9 Chemin de Bellevue, BP 110, 74941 Annecy-leVieux Cedex, France

12 Astroparticule et Cosmologie (APC), CNRS, Universite Paris 7 Denis Diderot, 10, rue Alice Domon et Leonie Duquet, 75205 Paris Cedex 13, France UMR 7164 (CNRS, Université Paris VII, CEA, Observatoire de Paris)

13 Dublin Institute for Advanced Studies, 5 Merrion Square, Dublin 2, Ireland

${ }^{14}$ Landessternwarte, Universität Heidelberg, Königstuhl, 69117 Heidelberg, Germany

15 Laboratoire de Physique Théorique et Astroparticules, CNRS/IN2P3, Université Montpellier II, CC 70, Place Eugène Bataillon, 34095 Montpellier Cedex 5, France

16 Universität Erlangen-Nürnberg, Physikalisches Institut, ErwinRommel-Str. 1, 91058 Erlangen, Germany

17 Laboratoire d'Astrophysique de Grenoble, INSU/CNRS, Université Joseph Fourier, BP 53, 38041 Grenoble Cedex 9, France

18 Institut für Astronomie und Astrophysik, Universität Tübingen, Sand 1, 72076 Tübingen, Germany

19 LPNHE, Université Pierre et Marie Curie Paris 6, Université Denis Diderot Paris 7, CNRS/IN2P3, 4 Place Jussieu, 75252 Paris Cedex 5, France

${ }^{20}$ Institute of Particle and Nuclear Physics, Charles University, V Holesovickach 2, 18000 Prague 8, Czech Republic

${ }^{21}$ Institut für Theoretische Physik, Lehrstuhl IV: Weltraum und Astrophysik, Ruhr-Universität Bochum, 44780 Bochum, Germany

${ }^{22}$ University of Namibia, Private Bag 13301, Windhoek, Namibia

23 Obserwatorium Astronomiczne, Uniwersytet Jagielloński, Kraków, Poland

${ }_{24}$ Nicolaus Copernicus Astronomical Center, Warsaw, Poland

25 School of Physics \& Astronomy, University of Leeds, Leeds LS2 9JT, UK

26 School of Chemistry \& Physics, University of Adelaide, Adelaide 5005, Australia

27 Toruń Centre for Astronomy, Nicolaus Copernicus University, Toruń, Poland

28 European Associated Laboratory for Gamma-Ray Astronomy, jointly supported by CNRS and MPG 